\title{
Vascular Age as a Cardiovascular Risk Marker in Asymptomatic Patients with Type 2 Diabetes
}

This article was published in the following Dove Press journal:

Diabetes, Metabolic Syndrome and Obesity: Targets and Therapy

\author{
Cátia Cristina \\ Silva Sousa Vergara Palma ${ }^{1}{ }^{1}$ \\ Pablo Moura Lopes ${ }^{2}$ \\ Eliete Leão Clemente Silva' \\ Maria de Fátima \\ da Matta Bevilaqua' \\ Alfredo \\ de Souza Bomfim² \\ Marilia Brito Gomes' \\ 'Department of Internal Medicine, \\ Diabetes Unit, State University Hospital \\ of Rio de Janeiro, Rio de Janeiro, Brasil; \\ ${ }^{2}$ Department of Internal Medicine, \\ Cardiology Unit, State University \\ Hospital of Rio de Janeiro, Rio de Janeiro, \\ Brasil
}

Aim: There is a wide variety of cardiovascular outcomes in patients with type 2 diabetes (T2DM), even in asymptomatic individuals. Carotid intima-media thickness (CIMT) is a marker of subclinical atherosclerosis and can be considered as a predictor of cardiovascular risk (CVR). The aim of this study was to evaluate the relationship between CIMT-determined vascular age (VA), CVR scores, and thyroid function in asymptomatic patients with T2DM. Patients and Methods: Clinical laboratory and CIMT parameters were measured in 154 asymptomatic patients with T2DM. The Framingham risk score (FRS) was performed with chronological age (CA) and with VA. A multinomial logistic regression model was used to analyze variables related to CVR reclassification.

Results: The use of CIMT-determined VA led to the reclassification of 54 (35.52\%) out of 152 asymptomatic T2DM patients, being $20(37.03 \%)$ to a lower categorical risk and 34 $(62.96 \%)$ to a higher categorical risk according to FRS. The variables that were associated to reclassification to a higher categorical risk were positive family history $(\mathrm{FH})$ of premature coronary artery disease $(p=0.046), F H$ of thyroid disease $(p=0.010)$, use of statins $(p=0.027)$, and free T4 levels $(\mathrm{p}=0.009)$.

Conclusion: VA determined from CIMT allowed the reclassification of the CVR in asymptomatic T2DM patients. FH of premature CAD, FH of known thyroid disease, use of statins, and free $\mathrm{T} 4$ levels were associated to a reclassification into a higher risk category. The use of doppler to perform CIMT measure is currently more accessible, especially in a low-middle income country like Brazil. However, further prospective studies must be performed to establish the predictive values of CIMT on atherosclerosis and how thyroid function acts like cardiovascular risk marker on CVR scores.

Keywords: vascular age, type 2 diabetes, cardiovascular risk, CIMT

\section{Introduction}

Patients with diabetes mellitus (DM) have a three to four times higher risk of suffering a cardiovascular event and two times higher mortality rates compared with the general population. ${ }^{1}$ However, there is heterogeneity in the classification of cardiovascular risk (CVR) in this group. There is a wide variety of cardiovascular outcomes in this population, even in asymptomatic individuals, who could benefit from strict clinical control and treatment goals.

Regarding cardiovascular exams, there is no consensus on which should be requested for the detection of subclinical coronary artery disease (CAD) in asymptomatic patients with T2DM. The 2018 recommendations of the American Diabetes Association (ADA) ${ }^{2}$ reinforce that routine screening for CAD for asymptomatic patients should not be performed. However, it recommends that classic risk factors
Correspondence: Cátia Cristina Silva Sousa Vergara Palma

Department of Internal Medicine, Diabetes Unit, Rio de Janeiro State University (UERJ), Boulevard 28 de Setembro, 77- $3^{\circ}$ Andar - Vila Isabel, Rio de Janeiro, RJ 2055I-030, Brazil

Tel +55 2128688224

Email catiasousapalma@gmail.com 
(hypertension, dyslipidemia, smoking, chronic kidney disease, albuminuria, and family history (FH)) of early CAD should be accessed annually and intensively treated. Despite the paradigm that aggressive treatment of risk factors for cardiovascular disease in DM is sufficient, the use of 10-year CVR stratification scores has greatly improved in the past decade and has enabled the reduction of morbidity and mortality in this population. ${ }^{3}$

Considering that CVR is multifactorial, the search for non-classical risk factors may contribute to the refinement of events prediction, especially in asymptomatic patients. Among non-traditional risk factors, thyroid function has assumed relevant importance due to its close relationship with heart function. Thyroid dysfunction, even if subclinical, has significant effects on cardiovascular function. ${ }^{4}$ It is recognized that both subclinical and clinical hypothyroidism, the two most prevalent thyroid dysfunctions, may be considered confounding factors for cardiovascular disease and metabolic syndrome. ${ }^{5}$ However, the mechanisms that may regulate progression to cardiovascular disease are not fully understood, especially in cases of subclinical hypothyroidism. ${ }^{6}$ TSH levels may also correlate directly with the carotid intima-media thickness (CIMT), an early marker of subclinical atherosclerosis in patients with subclinical hypothyroidism and overt hypothyroidism. ${ }^{7,9}$ Another study described ${ }^{9}$ that CIMT is independently associated with thyroid function even within the normal reference range, suggesting an increased CVR even in subjects with normal thyroid function.

The determination of vascular age (VA), which acts as a "window" in correspondence to the coronary arteries, has been shown to be an effective tool in the detection of preclinical coronary disease. ${ }^{10}$ Many clinical and epidemiologic trials that used CIMT-determined VA provided a chance to adjust a patient's chronological age (CA) into his atherosclerotic burden. ${ }^{11,12}$ The replacement of the patient's $\mathrm{CA}$ in the Framingham risk score to the VA corresponding to the 50th percentile of the CIMT is another possibility to better predict CVR in patients with and without DM. ${ }^{11}$ The use of these tools, for detecting and improving the accuracy of CVR independently of classically known risk factors should be reinforced because it could result in risk reclassification.

The aim of the study was to evaluate the relationship between CIMT-determined VA, CVR scores, and thyroid function in asymptomatic patients with type 2 diabetes (T2DM).

\section{Patients and Methods}

This was an observational, cross-sectional, single-center study with 154 consecutive patients with T2DM who regularly attended a tertiary diabetes outpatient unit at Policlinica Piquet Carneiro, State University of Rio de Janeiro (PPC/ UERJ), from March 2014 to December 2018. The study protocol was approved by the Institutional Research Ethics Committee, by the number CAAE:31940114.8.0000.5259.

The inclusion criteria were: duration of T2DM longer than 12 months, patients older than 18 years old, without symptomatic cardiac diseases (that means without previous history of coronary heart disease (CHD) or symptoms of CHD: angina, shortness of breath, and fatigue; without history of cerebrovascular disease or peripheral arterial disease), without insulin use in the first year after diagnosis, and without history of ketosis or ketonuria. The exclusion criteria were: positive response to the short version of the Rose angina questionnaire, ${ }^{13}$ previous history of atherothrombotic events, patients who were unable to understand and/or sign the informed consent form, pregnant women, lactating women, chronic alcoholism, congestive heart failure, severe acute or obstructive respiratory failure, infection, patients with previous thyroid diseases, patients with recent interventions such as pulse therapy with corticosteroids, radioiodine, use of amiodarone, diabetes-related acute complications and hospitalization within the last 6 months. Written informed consent for the study was obtained from all patients.

All patients underwent a clinical exam, anthropometric measurements, demographic survey, laboratory evaluation, and carotid ultrasound to determine CIMT. The following variables were assessed: gender, age, self-reported color, race, duration of DM, BMI, blood pressure (systolic and diastolic), smoking, and familiar history of (in first and second-degree relatives): T2DM, obesity, hypertension, premature coronary artery disease and thyroid diseases (hypothyroidism, hyperthyroidism, thyroid cancer and nodules). Data on comorbidities, such as hypertension (defined as the use of antihypertensives and/or BP> 140x90 in more than two measurements in sitting, lying, and standing position), dyslipidemia (defined as the use of statins), diabetic neuropathy, chronic renal disease, and diabetic retinopathy (DR) were also obtained. Blood samples were collected for biochemical analysis: glycemia, total cholesterol, triglycerides, HDL cholesterol, LDL cholesterol-all by enzymatic colorimetric method (A15 analyzer, BioSystems); HbA1c by high-performance liquid chromatography (D10, BioRad); and 
free thyroxine (FT4) and thyrotropin (TSH): all by electrochemiluminescence (Cobas Elecsys20/10, Roche Hitachi). The reference values and intra-assay and inter-assay coefficients of variation (\%) were, respectively: 0.93 to $1.7 \mathrm{ng} / \mathrm{dL}$, $1.8 \%$ and $3.0 \%$ for FT4; 0.27 to $4.20 \mu \mathrm{UI} / \mathrm{mL}$, and $1.9 \%$ and $2.2 \%$ for TSH. LDL cholesterol was estimated indirectly by use of Friedwald's formula when triglycerides were $\leq 400$ $\mathrm{mg} / \mathrm{dL}^{14}$

Renal function was estimated by the CKD-EPI equation ${ }^{15}$ and was expressed as glomerular filtration rate (GFR) in milliliters per minute per $1.73 \mathrm{~m}^{2}$ ( $\left.\mathrm{mL} / \mathrm{min}\right)$. We considered all patients as non-African- American in the CKD-EPI equation. Albuminuria analysis was performed in a random morning (spot). Patients were instructed to avoid physical activity before collecting the urine sample. Patients submitted to kidney transplantation and those with urinary infection or hematuria were excluded. This procedure was repeated 3 times with a minimum interval of one week between each collection and a maximum period of six months. Urine concentrations of creatinine and albumin were measured by immunoturbidimetry, and the results were expressed as $\mathrm{mg} /$ g. The albumin-creatinine ratio $>30 \mathrm{mg} / \mathrm{g}$ was considered altered. The mean of at least two samples was used for the diagnosis. Patients were divided into two groups, as having: Normal renal function or CKD. Patients with normal renal function had a GFR $\geq 60 \mathrm{~mL} / \mathrm{min}$ and the absence of albuminuria. CKD was defined as a GFR $<60 \mathrm{~mL} / \mathrm{min}$ and/or the presence of albuminuria. ${ }^{16}$

The screening for DR was performed by mydriatic binocular indirect ophthalmoscopy (BIO), which was performed by an experienced retinal specialist. The classification of DR for each patient was assessed for the eye with the highest level of commitment. Each eye was classified based on the absence or presence of DR. Regarding DR, patients were classified as: absent, non-proliferative diabetic retinopathy (NPDR), proliferative diabetic retinopathy (PDR) and macular edema, according to the international classification of DR. ${ }^{17}$

For the analysis of 10-year CVR in patients with T2DM, the Framingham risk score (FRS) was applied. ${ }^{18}$ As proposed in the Framingham study, we classified the patients as follows: with a CVD risk between: $0 \%$ to $6 \%$ as a low-risk group, $6 \%$ to $20 \%$ as moderate-risk group, and $>20 \%$ as a high-risk group. Regarding CVR, the criterion of percentage of risk variation was used.

Arbitrarily, we used the 5\% variation for men and 2.5\% for women both for increasing or decreasing risk when comparing the risk calculated by $\mathrm{CA}$ to the risk compared to vascular age.
A single observer performed CIMT images in a dark, silent, air-conditioned room after resting the patient for at least five minutes. The exams were performed in the morning, in a room with penumbral light to facilitate the visualization of the structures in the monitor. We used Philips Eco-Collor Doppler ultrasound, model iE33, with an S5-1 (1-5MHz) sector heart probe and L11-3Hz linear probe (3-11MHZ). For the measurement of the CIMT, we used the semi-automatic edge detection program QLAB Advanced Ultrasound Quantification Software version 7.1, also from Philips brand. 3.5.1. Transverse sweeping of the carotids and their extra-cranial branches was performed bilaterally in search of visible plaques. After this, with the transducer longitudinal to the carotid, the CIMT was measured bilaterally in the distal wall of the common carotid artery ten millimeters proximal of the bulb, in three different angulations: posterior, lateral, and anterior. The boundaries of the intima-media layer were defined from the edge of the first ultrasound signal in the lumen of the vessel to the edge of the second ultrasound signal between this layer and the tunica-albuginea. The mean of the measurements of the 3 angulations was used to determine the mean CIMT. All images were obtained in accordance with the recommendations of the American Society of Echocardiography. ${ }^{10}$

VA was determined according to the normality tables derived from the ARIC study. ${ }^{19}$ VA was determined as the age at which the mean CIMT value for each side (right and left) represented the median value (50th percentile) of its given ethnic group and gender. The maximum VA was considered 89.80 years for women and 82.74 years for men. These values were derived from the average life expectancy of Brazilian women and men, respectively, with addition of one decade. ${ }^{20}$ The greater VA was used in the occurrence of divergence between both sides. The $\mathrm{FRS}^{18}$ was used for the stratification of CVR. Initially, it was calculated using the CA and, later, with the greater VA calculated in substitution to the CA.

\section{Statistical Analysis}

Exploratory data analysis was performed at first. The continuous variables were described using means and standard deviation ((SD) or medians and interquartile range [IQR]) and numbers with relative frequencies for categorical variables. Normality of data was evaluated by KolmogorovSmirnov test. The variables without normal distribution were logarithmized. The chi-square test (X2) and Fisher's exact test were used to evaluate the relationship 
between categorical variables in groups. Comparisons between independent continuous variables were performed using independent $T$ test, ANOVA (with Sidak correction), and Mann-Whitney or Kruskal-Wallis when appropriate and between continuous dependent variables by paired $T$ test or Wilcoxon rank test, when indicated. Multinomial logistic regression model was used to analyze variables related to the categories of Framingham score risk reclassification. Through the exponentiation of the coefficients (betas), the relative risk ratio (RRR) was obtained. The RRR of a coefficient indicates how the risk of the outcome falling in the comparison group compared to the risk of the outcome falling in the referent group changes with the variable in question. ${ }^{21}$ Simple multinomial logistic regression models were adjusted for each independent variable, and those with a p-value of up to 0.20 were selected. In the multiple multinomial regression, all the variables selected in the previous step were taken. The variables, non-significant at 0.05 , were removed one by one; remaining in the final model were only the variables with p-values less than or equal to 0.05 in at least one of the categories. The regression models were adjusted using statistical software. Analyses were performed using SPSS version 24 (SPSS, Inc., Chicago, Illinois, USA) and statistical software R (R Core TEAM, 2016). A two-sided p-value less than 0.05 was considered significant.

\section{Results}

\section{Overview of Demographic and Laboratory Data of the Studied Population}

A total of 154 patients, $59.7 \%$ female, were enrolled. The overview of the studied population and clinical and laboratory characteristics are presented in Table 1.

\section{Overview of Demographic and Laboratory Data of the Studied Population Stratified by CVR with CA and VA}

Patients were stratified according to Framingham global risk score using CA and then VA into three groups: low, intermediate, and high risk. Only 152 patients underwent ultrasonography for CIMT measurement. The overview of this data is presented in supplementary Tables 1 and 2 .

The variables that presented clinically relevant differences among low-, medium-, and high-risk patients stratified by Framingham risk score with CA were:
Table I Clinical and Laboratory Characteristics of the Studied Population

\begin{tabular}{|c|c|}
\hline Data & $n=154$ \\
\hline \multicolumn{2}{|l|}{ Demographic data } \\
\hline Females, n (\%) & $92(59.7)$ \\
\hline Age (years) & $54.6 \pm 10.1$ \\
\hline Age at diagnosis (years) & $42.3 \pm 10.6$ \\
\hline Known duration of diabetes (years) & $12.6 \pm 8.3$ \\
\hline Years of school attendance & $9.4 \pm 4.3$ \\
\hline Self-reported color-race (White/non-White), n (\%) & $39.6 / 60.4$ \\
\hline \multicolumn{2}{|l|}{ Positive Family history (FH) } \\
\hline $\mathrm{FH}$ of type 2 diabetes, $\mathrm{n}(\%)$ & II5 (74.7) \\
\hline FH of obesity, n (\%) & $66(42.9)$ \\
\hline FH of hypertension, $n(\%)$ & $119(77.3)$ \\
\hline $\mathrm{FH}$ of premature CAD, n (\%) & $59(38.3)$ \\
\hline FH of known thyroid dysfunction, $n$ (\%) & $15(9.7)$ \\
\hline \multicolumn{2}{|l|}{ Anthropometric data } \\
\hline BMI (kg/m2) & $30.6 \pm 5.3$ \\
\hline $\mathrm{SBP}(\mathrm{mm} \mathrm{Hg})$ & $132.1 \pm 15.7$ \\
\hline DBP $(\mathrm{mm} \mathrm{Hg})$ & $78.8 \pm 9.7$ \\
\hline HR (bpm) & $81.1 \pm 13.3$ \\
\hline Waist (cm) & $99.5 \pm 11.6$ \\
\hline WHR $(\mathrm{cm})$ & $0.9 \pm 0.09$ \\
\hline \multicolumn{2}{|l|}{ Classic cardiovascular risk factors } \\
\hline Hypertension, n (\%) & $116(75.3)$ \\
\hline Dyslipidemia, n (\%) & $133(86.3)$ \\
\hline Current Smokers, n (\%) & $13(8.4)$ \\
\hline Sedentarism, n (\%) & $109(70.8)$ \\
\hline Current alcohol consumption, $\mathrm{n}(\%)$ & $63(59.1)$ \\
\hline \multicolumn{2}{|l|}{ Treatment data } \\
\hline Basal insulin, $\mathrm{n}(\%)$ & $85(55.6)$ \\
\hline Metformin, n (\%) & $145(94.8)$ \\
\hline Statins, n (\%) & $120(77.9)$ \\
\hline Antihypertensive drugs, $\mathrm{n}(\%)$ & $116(75.3)$ \\
\hline Aspirin, n (\%) & $53(35.3)$ \\
\hline \multicolumn{2}{|l|}{ Chronic diabetes-related complications } \\
\hline Retinopathy, yes, n (\%) & $51(33.6)$ \\
\hline CKD (GFR <60mL/min and/or albuminuria), $\mathrm{n}$ (\%) & $22(14.3)$ \\
\hline Neuropathy (self-reported ou symptoms), n (\%) & $75(48.7)$ \\
\hline \multicolumn{2}{|l|}{ Laboratory data } \\
\hline Fasting glucose (mg/dl) & $171.9 \pm 85.2$ \\
\hline HbAlc (\%) & $8.1 \pm 1.7$ \\
\hline Total cholesterol (mg/dl) & $194.2 \pm 61.7$ \\
\hline Triglycerides (mg/dl) & $162(4 \mid-820)$ \\
\hline HDLc (mg/dL) & $57.9 \pm 17.9$ \\
\hline LDLc (mg/dL) & $96.4 \pm 41.4$ \\
\hline $\mathrm{TSH}(\mu \mathrm{Ul} / \mathrm{mL})$ & $2.1 \pm 1.4$ \\
\hline FreeT4 (ng/dL) & $1.2 \pm 0.2$ \\
\hline CRP $(\mathrm{mg} / \mathrm{dl})$ & $0.5(0.01-4.2)$ \\
\hline
\end{tabular}

(Continued) 
Table I (Continued).

\begin{tabular}{|l|l|}
\hline Data & $\mathbf{n}=154$ \\
\hline Uric acid $(\mathrm{mg} / \mathrm{dL})$ & $6.2 \pm 2.2$ \\
Albumin $(\mathrm{g} / \mathrm{dL})$ & $4.7 \pm 0.97$ \\
Albuminuria $(\mathrm{mg} / \mathrm{g})$ & $9.8(0.2-374.1)$ \\
GFR $(\mathrm{mL} / \mathrm{min} / \mathrm{l} .73 \mathrm{~m} 2)$ & $85.6 \pm 25$. \\
\hline Intima-media thickness data* & \\
IMT on the right (mm) & $0.66 \pm 0.14$ \\
IMT on the left (mm) & $0.66 \pm 0.13$ \\
IMT on worst side (mm) & $0.70 \pm 0.14$ \\
Vascular age using right CIMT (years) & $54.8 \pm 13.9$ \\
Vascular age using left CIMT (years) & $54.1 \pm 13.5$ \\
Vascular age using worst side & $58.6 \pm 14.0$ \\
\hline Framingham risk score & \\
FRS & $19.5 \pm 13.3$ \\
FRS with VA on worst side & $22.3 \pm 17.2$ \\
\hline
\end{tabular}

Notes: Data are presented as mean $\pm \mathrm{SD}$ or in median and interquartile range [IQR]. *IMT was obtained in 152 patients.

Abbreviations: N, number; $\mathrm{FH}$, family history; $\mathrm{CAD}$, coronary artery disease; $\mathrm{BMI}$, body mass index; SBP, systolic blood pressure; DBP, diastolic blood pressure; HR, heart rate; WHR, waist-to-hip ratio; CRD, chronic renal disease AAS, acetylsalicylic acid; GFR, glomerular filtration rate calculated by CKD-EPI formula; CKD, chronic kidney disease HDL, high density lipoprotein; LDL, low density lipoprotein; TSH, thyroid-stimulating hormone; Free T4, free thyroxine hormone CRP, C-reactive protein; IMT, intima media thickness; FRS, Framingham risk score; VA, vascular age.

WHR, statins use, free T4, triglycerides, albumin, GFR, and uric acid. The comparison between groups after corrections for multiple comparisons showed that WHR and triglycerides presented differences between intermediate- and high-risk groups $(\mathrm{p}=0.031$ and 0.002 , respectively); GFR and uric acid remained different among the three risk groups; and albumin was different between low- and high-risk groups $(p=0.029)$. Free T4 did not reach a significance level for multiple comparisons $(\mathrm{p}=0.07)$. Use of statins was different between low- and intermediate-risk groups $(\mathrm{p}=0.001)$.

The variables that presented differences among low-, medium-, and high-risk patients stratified by Framingham risk score with VA were: HbA1c\%, triglycerides, GFR and uric acid. The comparison between groups after correction for multiple comparisons showed that uric acid and triglycerides presented differences between the intermediate- and high-risk groups $(p=0.008$ and $p=0.013$, respectively); GFR was different between low- and highrisk groups $(\mathrm{p}=0.011)$; and $\mathrm{HbA} 1 \mathrm{c} \%$ did not reach a significance level for multiple comparisons $(\mathrm{p}=0.072)$. The FH of obesity, hypertension and DM were not different between groups.
Overview of Demographic and Laboratory Data of the Studied Population After Reclassification When Using Both Scores Ninety-eight out of $152(64.5 \%)$ patients had CVR concordant in both scores $(\mathrm{n}=7(7.1 \%)$ in low risk, $\mathrm{n}=55(56.1 \%)$ in intermediary risk, $n=36(36.7 \%)$ in high risk; $p<0.001)$. A total of $54(35.5 \%)$ patients in all risk categories were reclassified. Only 15 patients had IMT $>0.9 \mathrm{~mm}(9.8 \%)$. Almost all patients $(94.8 \%)$ used metformin as a treatment for type 2 diabetes without difference among groups $(\mathrm{p}=0.573)$.

Twenty (13.2\%) patients had the risk reduced, and $34(22.4 \%)$ had the risk increased after the replacement of CA by VA in the score. Among the 14 patients who were in the low-risk category, seven $(50 \%)$ were reclassified to intermediate-risk. Of the eighty-nine patients who were in the intermediate-risk category, seven were reclassified to the low-risk category, and 27 were reclassified to the high-risk category. Among the 51 patients in the high-risk category, 13 were reclassified to intermediate risk. No patient in this group was classified as low- risk.

Thirty women (32.9\%) had their risk reclassified: 21 (70\%) reclassified to a higher risk class and nine (30\%) for a lower risk class. Among men, 24 (39.3\%) had their risk reclassified: $13(54.2 \%)$ reclassified to a higher risk class, and $11(45.8 \%)$ for a lower risk class. We did not observe difference between genders in the reclassification of the risk $(\mathrm{p}=0.26)$.

The median of the difference between VA and CA of male and female (median and interquartile range [IQR] was similar: 3.44 ( -24.11 to 30.00 ) and 2.43 ( -21.57 to 42.80$)$ ), respectively. Regarding the percentage of variation in CVR, we observed that $98(64.5 \%)$ of all the patients presented this variation for both increased and decreased CVR. Males presented percentage of risk variation of $27 \%(\mathrm{n}=41)$ and females a percentage of risk variation of $37.6 \%(n=57)$, without difference between gender.

\section{Overview of Demographic and Laboratory Data of the Studied Population Considering Risk of Changing Category}

Patients were divided into three groups according to risk of category change: maintenance, increased, and decreased. The maintenance group $n=98(64.5 \%)$ included the patients who had concordant risk in both scores. The decreased risk group $n=20(13.1 \%)$ included patients who 
had the risk reduced: intermediate to low or high to intermediate. The increased risk group $n=34$ (22.4\%) included patients who had increased the risk from low to intermediate or intermediate to high. No socio-demographic, clinical or laboratory variables were associated with risk change. The overview of this data is presented in Supplementary Table 3.

To analyze variables related to the different categories of CVR reclassification, the multinomial logistic regression model was used. A simple multinomial logistic regression was performed with all variables at first. The results are shown in Table 2.

The variables selected for the final model were: FH for premature $\mathrm{CAD}, \mathrm{FH}$ of known thyroid disease, retinopathy, use of statins, free T4, albuminuria, and sedentary lifestyle. The final model is demonstrated in Table 3.

\section{Discussion}

Our study carried on in a low-middle income country and in an admixed population, demonstrated that the use of CIMT for the calculation of VA allowed the reclassification of risk in 54 (35.5\%) of the 152 asymptomatic patients with T2DM in all risk categories. Reclassification to a higher risk group was associated with FH of premature CAD, FH for thyroid disease, use of statins, and free T4. None of the variables associated with glycemic control and diabetes-related chronic complications influenced the reclassification of CVR.

The CIMT can be considered as a surrogate marker of atherosclerosis and is widely used to improve accuracy in the stratification of CVR. ${ }^{22}$ It is a non-invasive and easily performed technique compared to other techniques used to

Table 2 Simple Multinomial Logistic Regression

\begin{tabular}{|c|c|c|c|c|c|c|}
\hline & \multicolumn{3}{|c|}{ Increase } & \multicolumn{3}{|c|}{ Decrease } \\
\hline & RRR & $95 \% \mathrm{Cl}$ & $\mathbf{p}$ & RRR & $95 \% \mathrm{Cl}$ & $\mathbf{p}$ \\
\hline \multicolumn{7}{|l|}{ Demographic data } \\
\hline Known duration of diabetes (years) & 0.97 & $0.92-1.02$ & 0.22 & 0.98 & $0.93-1.04$ & 0.58 \\
\hline Years of school attendance & 0.96 & $0.87-1.06$ & 0.40 & 0.95 & $0.84-1.07$ & 0.42 \\
\hline FH of known TD & 3.37 & $1.09-10.46$ & 0.03 & 0.68 & $0.08-5.89$ & 0.73 \\
\hline $\mathrm{FH}$ of premature CAD & 1.78 & $0.8 \mathrm{I}-3.90$ & 0.15 & 0.28 & $0.08-1.02$ & 0.05 \\
\hline Retinopathy & 1.84 & $0.75-4.52$ & 0.18 & 1.10 & $0.0-4.30$ & 0.86 \\
\hline \multicolumn{7}{|l|}{ Anthropometric data } \\
\hline BMI $(\mathrm{kg} / \mathrm{m} 2)$ & I & $0.92-1.08$ & 0.94 & 1.03 & $0.94-1.13$ & 0.58 \\
\hline Waist $(\mathrm{cm})$ & I & $0.97-1.04$ & 0.92 & 1.02 & $0.98-1.07$ & 0.39 \\
\hline WHR $(\mathrm{cm})$ & 2.64 & $0.05-147.77$ & 0.63 & 13.39 & $0.15-1209.04$ & 0.26 \\
\hline \multicolumn{7}{|l|}{ Classical CV risk factors } \\
\hline Sedentary lifestyle & 0.77 & $0.34-1.76$ & 0.54 & 2.38 & $0.65-8.75$ & 0.19 \\
\hline \multicolumn{7}{|l|}{ Treatment data } \\
\hline Aspirin & 1.56 & $0.65-3.72$ & 0.32 & 0.71 & $0.27-1.89$ & 0.49 \\
\hline Statins & 2.47 & $1.1-6.08$ & 0.05 & 1.23 & $0.36-4.16$ & 0.73 \\
\hline Basal insulin & 0.82 & $0.38-1.81$ & 0.63 & 0.73 & $0.28-1.92$ & 0.52 \\
\hline \multicolumn{7}{|l|}{ Laboratory data } \\
\hline CRP $(\mathrm{mg} / \mathrm{dl})$ & 0.72 & $0.33-1.54$ & 0.36 & 1.27 & $0.67-2.4$ & 0.46 \\
\hline $\mathrm{HbAlc} \%$ & 1.06 & $0.84-1.33$ & 0.62 & 1.01 & $0.76-1.35$ & 0.94 \\
\hline $\mathrm{TSH}(\mu \mathrm{Ul} / \mathrm{mL})$ & 0.99 & $0.74-1.33$ & 0.97 & 1.03 & $0.73-1.46$ & 0.85 \\
\hline FreeT4 (ng/dL) & 0.07 & $0.01-0.79$ & 0.03 & 2.79 & $0.16-48.97$ & 0.48 \\
\hline Triglycerides (mg/dl) & I & 1 & 0.32 & I & $1.0-1.0$ & 0.98 \\
\hline Albumin (g/dL) & 0.82 & $0.53-1.26$ & 0.36 & 0.93 & $0.56-1.53$ & 0.76 \\
\hline GFR $(\mathrm{mL} / \mathrm{min} / 1.73 \mathrm{~m} 2)$ & 1.01 & $0.99-1.02$ & 0.46 & I & $0.98-1.02$ & 0.81 \\
\hline Uric acid (mg/dl) & 0.98 & $0.82-1.17$ & 0.82 & 1.11 & $0.91-1.36$ & 0.32 \\
\hline Albuminuria (mg/g) & 1.01 & $1.0-1.01$ & 0.02 & 1.01 & $1-1.01$ & 0.12 \\
\hline
\end{tabular}

Note: Data are presented in mean $\pm S D$ or in median and interquartile range [IQR].

Abbreviations: $\mathrm{FH}$, family history; TD, thyroid disease; CAD, coronary artery disease; BMI, body mass index; WHR, waist-to-hip ratio; CV, cardiovascular; CRP, C-reactive protein; TSH, thyroid-stimulating hormone; Free T4, free thyroxine hormone; GFR, glomerular filtration rate calculated by CKD-EPI formula; IMT, intima-media thickness. 
Table 3 Multiple Multinomial Logistic Regression- Final Model

\begin{tabular}{|c|c|c|c|c|c|c|c|c|}
\hline & \multicolumn{3}{|c|}{ Increase } & \multirow[t]{2}{*}{$\mathbf{p}$} & \multicolumn{3}{|c|}{ Decrease } & \multirow[t]{2}{*}{$\mathbf{p}$} \\
\hline & Beta & RRR & $95 \% \mathrm{Cl}$ & & Beta & RRR & $95 \% \mathrm{Cl}$ & \\
\hline $\mathrm{FH}$ of premature CAD & 0.892 & 2.44 & $1.012-5.88 \mid$ & 0.046 & -1.421 & $0.24 I$ & $0.063-0.913$ & 0.036 \\
\hline FH of known TD & 1.656 & 5.243 & $1.480-18.563$ & 0.010 & -0.551 & 0.576 & $0.062-5.307$ & 0.626 \\
\hline Statins & 1.086 & 2.964 & I.128-7.792 & 0.027 & 0.291 & 1.338 & $0.383-4.669$ & 0.647 \\
\hline Free T4 & -3.434 & 0.032 & $0.002-0.432$ & 0.009 & 1.935 & 6.928 & $0.289-166.02$ & 0.232 \\
\hline
\end{tabular}

Abbreviations: F, family history; TD, thyroid disease; CAD, coronary artery disease; Free T4, free thyroxine hormone.

identify subclinical atherosclerosis. In view of the heterogeneity of patients with T2DM, a wide variety of cardiovascular outcomes is observed. VA, also known as heart age, arterial age, or CVR age, is a concept that supposes that the conversion of $\mathrm{CA}$ to VA derived from vascular imaging data would lead to a refinement of individual CVR. ${ }^{23}$ Although our study is cross-sectional, reclassifying patients into a higher risk category using VA, demonstrates an improvement of risk stratification and possibly a more precise treatment in routine clinical practice. The refinement of CVR perdition using VA can be overserved in patients with and without DM. The study of Adolphe et $\mathrm{al}^{23}$ where the use of VA allowed to change CVR prediction in multi-ethnic patients, and Greenland ${ }^{24}$ have shown that the use of VA derived from intima-media thickness (IMT) promotes a 4-5-fold increase in CVR and cerebrovascular events in relation to classical coronary risk in subjects without DM. They considered VA to be a more relevant biological measure than CA. Previous studies ${ }^{25,26}$ performed in patients with T2DM, with populations derived from own framingham cohort, caucasians and only one study with the Japanese population demonstrated that the combination of the Framingham score and IMT provided greater predictive power of cardiovascular events compared to the use of Framingham risk score alone, including asymptomatic patients. In these studies, the increase in CIMT was associated with a higher incidence of cardiovascular events in the background populations and in patients with DM. Even in patients with type 1 diabetes, the use of VA age may contribute to the identification of high CVR, ${ }^{27}$ although the course of cardiovascular disease is different when compared to T2DM.

However, there are controversies in studies that use CIMT for detection of subclinical atherosclerosis. In the meta-analysis of USE-IMT, ${ }^{28}$ the authors did not observe improvement in accuracy in risk prediction in patients with DM when measurement of the mean common CIMT was added to the Framingham risk score. However, they point out that there were some limitations of their study, such as: definition of DM, duration, severity of the disease, and use of medications in the various cohorts analyzed. In the study of Yeboah et $\mathrm{al}^{29}$ combining data from T2DM without clinical CVD in the Multi-Ethnic Study of Atherosclerosis (MESA) and in the Heinz Nixdorf Recall Study, ${ }^{30}$ the CIMT was not an independent predictor for CHD risk estimator, but this fact was noted with coronary calcium calcification (CAC). CAC was able to best predict CHD compared to other subclinical markers in T2DM.

One point to consider is about performing cardiovascular tests in evaluation of patients with DM. The Brazilian Guidelines for the Prevention of Cardiovascular Disease in Diabetes $2017^{31}$ recommends that the resting ECG should be performed annually in all patients with $\mathrm{DM}$, even asymptomatic, due to its low cost and risk. In our study, we identified in $8.1 \%$ of patients the presence of electrocardiographic changes suggestive of ischemic coronary disease. Even all patients being asymptomatic and responded negatively to the Rose questionnaire. These patients had a median of IMT $0.806 \mathrm{~mm}$ and were classified as high-risk group according to the Framingham scores. (data not shown).

Our study demonstrated that positive $\mathrm{FH}$ of premature CAD was associated with reclassification of the patients to a higher risk class. These data are in agreement with the study by Wang et al, ${ }^{32}$ which showed that subclinical atherosclerosis, assessed in the carotid arteries, was more prevalent in individuals with a family history of CHD. Parental CHD at a premature age can identify a subgroup with a strong familial cluster to vascular diseases ${ }^{33}$ According to the ASE consensus, ${ }^{22}$ patients with an $\mathrm{FH}$ of premature CAD should also be considered for carotid ultrasound for IMT measurement and plaque detection.

Another association was with $\mathrm{FH}$ of thyroid disease, although less prevalent, was associated with changing to a higher risk class. Nevertheless, considering the amplitude of the confidence interval and the missing relevant data in 
the literature to corroborate this association, more studies are needed to assess the influence of $\mathrm{FH}$ of thyroid disease in the stratification of CVR. The FH of thyroid disease is best documented in cases of differentiated thyroid cancer (DTC), ${ }^{34}$ which are mainly sporadic. In this condition, FH of thyroid disease is also considered a risk factor for familial tumors and may account for $5 \%$ to $15 \%$ of the cases. ${ }^{34}$ In spite of that, it is important to emphasize that both $\mathrm{FH}$ of $\mathrm{CHD}$ and $\mathrm{FH}$ of thyroid disease were selfreported.

The increase in free T4 within the normal range was associated with a lower chance of classification in the riskincreasing group. This was an unexpected result, since the increase in free T4 was not associated with a change in TSH levels. Compared with serum TSH levels, both free $\mathrm{T} 3$ and free T4 are less sensitive and specific in the diagnosis of thyroid disorders. Single measurements of FT3 and FT4 serum are unable to diagnose subclinical thyroid disorders.

Considering the thyroid function, almost $6 \%$ of our study population presented subclinical hypothyroidism, even considering the exclusion criteria of the study (data not shown). However, none presented TSH values higher than $10 \mathrm{mUI} / \mathrm{L}$, which is related to the increased risk of CAD, according to previous studies. ${ }^{35,36}$ In view of patients with T2DM with no evidence of thyroid disease, Giandalia et $\mathrm{al}^{37}$ demonstrated that higher values of TSH within normal range were more frequent in women and younger patients and were associated with visceral obesity and higher triglyceride concentrations - two well-known CVD risk factors, although in this study, no $\mathrm{CV}$ outcomes were evaluated. Considering CIMT and thyroid function, in the Elsa Brazil study, ${ }^{38}$ after a multivariate adjustment for sociodemographic and CVR factors, subclinical hypothyroidism was associated with higher CIMT values using euthyroid subjects as the reference. However, in other studies, the authors observed that thyroid hormone was not predictive of carotid atherosclerosis when adjusted for confounders, ${ }^{39}$ and no significant relationship between CIMT and serum TSH levels was observed in normal, nonthyroxine-taking subjects. ${ }^{40}$ More studies should be carried out to evaluate the association between subclinical thyroid dysfunction and subclinical atherosclerosis measured by CIMT.

Despite not having entered the final model, possibly due to the large number of patients without or with mild retinopathy in our study, DR, mainly PDR and NPDR, severe, or moderate, has been considered a predictor of risk for CVD in patients with $\mathrm{DM}^{41}$

The use of statins in our study was related to risk of reclassification to a higher class of CVR. Although controversial, the use of statins suggests that these patients already have an increased CVR. Even though, in one study, ${ }^{34}$ the authors demonstrated that each $10 \%$ reduction in LDL-C was estimated to reduce IMT by $0.73 \%$ per year $(95 \% \mathrm{CI}$, 0.27 to 1.19 ), the use of statins was not sufficient to prevent the reclassification to a higher cardiovascular risk group in our population of patients with asymptomatic T2DM. However, it is important to note that only $40.3 \%$ of patients were within the recommended LDL goal for high-risk patients according to the current Brazilian Cardiovascular Risk Prevention guidelines. ${ }^{31}$

Some limitations of this study should be mentioned. The patients were recruited into a single tertiary care center with only internal validity. Only one TSH/Free T4 sample was collected for the determination of thyroid function, as has been done in other studies evaluating thyroid function ${ }^{38}$ and the determination of antiperoxidase antibodies was not performed. Another potential limitation was the reliance on self-reported $\mathrm{FH}$ of premature CAD and of thyroid disease, although most studies addressed this in the same way. ${ }^{34}$ The variation of CVR in 5\% for men and $2.5 \%$ for women can also be considered a limitation because, no data in the literature to validate this variation were found. Furthermore, considering that our study was a cross-sectional one, the causal relationship among the studied variables and CVR estimated by CA or VA could not be established. No other noninvasive method was used to compare VA with CA.

\section{Conclusion}

In our study, the use of CIMT to determine VA allows the reclassification of the CVR risk in asymptomatic T2DM patients. The predicted variables associated to reclassification to a higher risk category were: $\mathrm{FH}$ of premature $\mathrm{CAD}$, FH of known thyroid disease, use of statins, and free T4. The use of doppler to perform CIMT measure is currently more accessible than other methods, such as coronary calcium score, myocardial scintigraphy or angio tomography of coronary arteries that are in general more expensive and invasive. This fact is an important issue, especially in a lowmiddle income country like Brazil, where less invasive and low-cost techniques should be encouraged, and possibly incorporated into the routine clinical practice. This study also adds the importance of using intima-media thickness to 
the Framingham risk score to improve the accuracy of risk stratification in patients with asymptomatic patients with T2DM in an admixed population. However, further prospective studies must be performed to establish the predictive values of CIMT as a subclinical marker of atherosclerosis on cardiovascular outcomes and evaluate how thyroid function acts like cardiovascular risk marker on CVR score of asymptomatic patients with T2DM.

\section{Acknowledgments}

The abstract of this paper was presented at the 22nd Brazilian Diabetes Society Congress as a poster presentation with interim findings. The author's reports that this study was conducted in accordance with the Declaration of Helsinki.

\section{Disclosure}

The author's reports no conflicts of interest in this work.

\section{References}

1. Haffner SM, Lehto S, Rönnemaa T, Pyörälä K, Laakso M. Mortality from coronary heart disease in subjects with type 2 diabetes and in nondiabetic subjects with and without prior myocardial infarction. $N$ Engl J Med. 1998;339(4):229-234. doi:10.1056/NEJM199807233390404

2. American Diabetes Association. Cardiovascular disease and risk management: standards of medical care in diabetes-2018. Diabetes Care. 2018;41(Supplement 1):S86-S104. doi:10.2337/dc18-S009.

3. Gæde P, Lund-Andersen H, Parving HH, Pedersen O. Effect of a multifactorial intervention on mortality in type 2 diabetes. $N$ Engl $J$ Med. 2008;58(6):580-591.

4. Klein I, Danzi S. Thyroid disease and the heart. Circulation. 2007;116:1725-1735.

5. Fox CS, Golden SH, Anderson C, et al. Update on prevention of cardiovascular disease in adults with type 2 diabetes mellitus in light of recent evidence: a scientific statement from the American Heart Association and the American Diabetes Association. Circulation. 2015;132(8):691-718. doi:10.1161/CIR.0000000000000230

6. National Institutes of H E. Expert panel on detection, evaluation, and treatment of high blood cholesterol program in adults; 2012. Available from: http://www.molinahealthcare.com/providers/wa/medicaid/ resource/PDF/health_wa_CholesterolCPG.pdf. Accessed March 28, 2016.

7. Nagasaki T, Inaba M, Henmi $Y$, et al. Decrease in carotid intimamedia thickness in hypothyroid patients after normalization of thyroid function. Clin Endocrinol (Oxf). 2003;59(5):607-612. doi:10.1046/j.1365-2265.2003.01893.x

8. Nagasaki T, Inaba M, Henmi Y, et al. Change in von Willebrand factor and carotid intima-media thickness in hypothyroid patients with normal thyroid function after levothyroxine replacement therapy. Eur $J$ Endocrinol. 2004;150(2):125-131. doi:10.1530/eje.0.1500125

9. Takamura N, Akilzhanova A, Hayashida N, et al. Thyroid function is associated with carotid intima-media thickness in euthyroid subjects. Atherosclerosis. 2009;204(2):e77-e81. doi:10.1016/j.atherosclerosis. 2008.09.022

10. Stein JH, Korcarz CE, Post WS. Use of Carotid Ultrasound to Identify Subclinical Vascular Disease and Evaluate Cardiovascular Disease Risk: summary and Discussion of the American Society of Echocardiography Consensus Statement. Prev Cardiol. 2009;12 (1):34-38. doi:10.1111/j.1751-7141.2008.00021.x
11. Ashrafian H, Lim TK, Senior R, et al. Carotid ultrasound imaging: an effective technique for detecting early atherosclerosis-vascular screening for cardiovascular disease has come of age. $J \mathrm{Am}$ Soc Echocardiogr. 2007;20(issue 1):1-3. doi:10.1016/j.echo.2006.06.016

12. Gepner AD, Keevil JG, Wyman RA, et al. Use of carotid intimamedia thickness and vascular age to modify cardiovascular risk prediction. J Am Soc Echocardiogr. 2006;19:1170-1174. doi:10.1016/j. echo.2006.04.009

13. Bastos M-S, Lotufo PA, Whitaker AL, Bensenor IM. Validation of the short-version of Rose angina questionnaire in Brazil. Arq Bras Cardiol. 2012;99(5):1056-1059. doi:10.1590/S0066-782X2012001400012

14. Friedewald WT, Levy RI, Fredrickson DS. Estimation of the concentration of low-density lipoproteincholesteroiln plasma, without use of the preparative ultracentrifuge. Clin Chem. 1972;18(6):499-502.

15. Levey AS, Stevens LA, Schmid $\mathrm{CH}$, et al. A new equation to estimate glomerular filtration rate. Ann Intern Med. 2009;150 (9):604-612.

16. Canadian Society of Nefrology. KDIGO 2012 Clinical Practice Guideline for the Evaluation and Management of Chronic Kidney Disease; 2012.

17. Wilkinson CP, Ferris FL 3rd, Klein RE. Proposed international clinical diabetic retinopathy and diabetic macular edema disease severity scales. Ophthalmology. 2003;110(9):1677-1682. doi:10.1016/S01616420(03)00475-5

18. D'Agostino RB, Vasan RS, Pencina MJ, et al. General cardiovascular risk profile for use in primary care: the Framingham heart study. Circulation. 2008;117(6):743-753. doi:10.1161/CIRCULATIONAHA.107.699579

19. Howard G, Sharrett AR, Heiss G, et al. Carotid artery intimal-medial thickness distribution in general populations as evaluated by B-mode ultrasound. ARIC Investigators. Stroke. 1993;24(9):1297-1304. doi:10.1161/01.STR.24.9.1297

20. IBGE. Projections and evaluation of the population of Brazil and Federation Units; August 7, 2018. Available from: https://www.ibge. gov.br/apps/populacao/projecao/. Accessed June 23, 2020.

21. UCLA. Multinomial logistic regression. Stata data analysis examples; May 23, 2019. Available from: https://stats.idre.ucla.edu/stata/dae/ multinomiallogistic-regression/. Accessed June 23, 2020.

22. Stein JH, Korcarz CE, Hurst RT, et al. Use of Carotid Ultrasound to Identify Subclinical Vascular Disease and Evaluate Cardiovascular Disease Risk: A Consensus Statement from the American Society of Echocardiography Carotid Intima-Media Thickness Task Force Endorsed by the Society for Vascular Medicine. J Am Soc Echocardiogr. 2008;21(2):93-111. doi:10.1016/j.echo.2007.11.011

23. Adolphe AB, Huang X, Cook LS. Carotid intima-media thickness determined vascular age and the Framingham risk score. Crit Pathw Cardiol. 2011;10(4):173-179. doi:10.1097/HPC.0b013e31823a31f0

24. Greenland P, Smith Jr SC Jr, Grundy SM. Improving coronary heart disease risk assessment in asymptomatic people: role of traditional risk factors and noninvasive cardiovascular tests. Circulation. 2001;104(15):1863-1867. doi:10.1161/hc4201.097189

25. Yoshida M, Mita T, Yamamoto $R$, et al. Combination of the Framingham risk score and carotid intima-media thickness improves the prediction of cardiovascular events in patients with type 2 diabetes. Diabetes Care. 2012;35(1):178-180. doi:10.2337/dc11-1333

26. Bernard S, Serusclat A, Targe F, et al. Incremental predictive value of carotid ultrasonography in the assessment of coronary risk in a cohort of asymptomatic Type 2 diabetic subjects. Diabetes Care. 2005;28 (5):1158-1162. doi:10.2337/diacare.28.5.1158

27. de Andrade CRM, Silva ELC, da Matta MDFB, Castier MB, Rosa MLG, Gomes MB. Vascular or chronological age: which is the better marker to estimate the cardiovascular risk in patients with type 1 diabetes? Acta Diabetol. 2016;53(6):925-933. doi:10.1007/s00592-016-0891-8

28. den Ruijter HM, Peters SAE, Groenewegen KA, et al. Common carotid intima-media thickness does not add to Framingham risk score in individuals with diabetes mellitus: the USE-IMT initiative. Diabetologia. 2013;56(7):1494-1502. doi:10.1007/s00125-013-2898-9 
29. Yeboah J, Erbel R, Delaney JC, et al. Development of a new diabetes risk prediction tool for incident coronary heart disease events: the multiethnic study of atherosclerosis and the Heinz Nixdorf recall study. Atherosclerosis. 2014;236(2):411-417. doi:10.1016/j.atherosclerosis. 2014.07.035

30. Mahabadi AA, Möhlenkamp S, Moebus S. The Heinz Nixdorf recall study and its potential impact on the adoption of atherosclerosis imaging in European primary prevention guidelines. Curr Atheroscler Rep. 2011;13(5):367-372. doi:10.1007/s11883-011-0199-7

31. Bertoluci MC, Moreira RO, Faludi A, et al. Brazilian guidelines on prevention of cardiovascular disease in patients with diabetes: a position statement from the Brazilian Diabetes Society (SBD), the Brazilian Cardiology Society (SBC) and the Brazilian Endocrinology and Metabolism Society (SBEM). Diabetol Metab Syndr. 2017;9(1). doi:10.1186/s13098-017-0251-Z

32. Wang TJ, Nam B-H, D’Agostino RB, et al. Carotid intima-media thickness is associated with premature parental coronary heart disease: the Framingham heart study. Circulation. 2003;108(5):572-576. doi:10.1161/01.CIR.0000081764.35431.DE

33. Expert Panel on Detection, Evaluation, and Treatment of High Blood Cholesterol in Adults. Executive Summary of the Third Report of the National Cholesterol Education Program (NCEP) Expert Panel on Detection, Evaluation, and Treatment of High Blood Cholesterol in Adults (Adult Treatment Panel III). JAMA. 2001;285(19):2486-2497. doi:10.1001/jama.285.19.2486.

34. Kust D, Staničić J, Mateša N. Bethesda thyroid categories and family history of thyroid disease. Clin Endocrinol (Oxf). 2018;88(3):468472. doi:10.1111/cen.13538
35. Walsh JP, Bremner AP, Bulsara MK, et al. Subclinical thyroid dysfunction as a risk factor for cardiovascular disease. Arch Intern Med. 2005;165(21):2467. doi:10.1001/archinte.165.21.2467

36. Rodondi N, den Elzen WPJ, Bauer DC, et al. Subclinical hypothyroidism and the risk of coronary heart disease and mortality. JAMA. 2010;304(12):1365. doi:10.1001/jama.2010.1361

37. Giandalia A, Russo GT, Romeo EL, et al. Influence of high-normal serum TSH levels on major cardiovascular risk factors and visceral adiposity Index in euthyroid type 2 diabetic subjects. Endocrine. 2014;47(1):152-160. doi:10.1007/s12020-013-0137-2

38. Bensenor IM. Thyroid disorders in Brazil: the contribution of the Brazilian Longitudinal Study of Adult Health (ELSA-Brasil). Braz J Med Biol Res. 2019;52(2). doi:10.1590/1414-431x20198417

39. Delitala AP, Filigheddu F, Orrù M, et al. No evidence of association between subclinical thyroid disorders and common carotid intima medial thickness or atherosclerotic plaque. Nutr Metab Cardiovasc Dis. 2015;25(12):1104-1110. doi:10.1016/j.numecd.2015.09.001

40. Jorde R, Joakimsen O, Stensland E, Mathiesen EB. Lack of significant association between intima-media thickness in the carotid artery and serum TSH level. The Tromsø study. Thyroid. 2008;18(1):21-25. doi:10.1089/thy.2007.0165

41. Avogaro A, Fadini GP. Microvascular complications in diabetes: A growing concern for cardiologists. Int J Cardiol. 2019;291:29-35. doi:10.1016/j.ijcard.2019.02.030

\section{Publish your work in this journal}

Diabetes, Metabolic Syndrome and Obesity: Targets and Therapy is an international, peer-reviewed open-access journal committed to the rapid publication of the latest laboratory and clinical findings in the fields of diabetes, metabolic syndrome and obesity research. Original research, review, case reports, hypothesis formation, expert opinion and commentaries are all considered for publication. The manuscript management system is completely online and includes a very quick and fair peer-review system, which is all easy to use. Visit http://www.dovepress.com/testimonials.php to read real quotes from published authors.

Submit your manuscript here: https://www.dovepress.com/diabetes-metabolic-syndrome-and-obesity-targets-and-therapy-journal 